\title{
Mesenchymal Stem Cells Decrease Oxidative Stress in the Bowels of Interleukin-10 Knockout Mice
}

Kyong Jin Jung ${ }^{1,2}$, Gun Woo Lee ${ }^{3}$, Chul Hyun Park ${ }^{3}$, Tae Jin Lee ${ }^{1}$, Joo Young Kim ${ }^{1}$, Eon Gi Sung ${ }^{1}$, Seong Yong Kim ${ }^{4}$, Byung Ik Jang ${ }^{5}$, and In Hwan Song ${ }^{1}$

${ }^{1}$ Department of Anatomy and ${ }^{2}$ Smart-Aging Convergence Research Center, Departments of ${ }^{3}$ Orthopedic Surgery, ${ }^{4}$ Biochemistry and Molecular Medicine, and ${ }^{5}$ Internal Medicine, Yeungnam University College of Medicine, Daegu, Korea

Background/Aims: Inflammatory bowel disease (IBD) is an autoimmune disease characterized by chronic inflammation mainly in the large intestine. The interleukin-10 knockout (IL-10 KO) mouse is a well-known animal model of IBD that develops spontaneous intestinal inflammation resembling Crohn's disease. Oxidative stress is considered to be the leading cause of cell and tissue damage. Reactive oxygen species (ROS) can cause direct cell injury and/or indirect cell injury by inducing the secretion of cytokines from damaged cells. This study evaluated the effects of mesenchymal stem cell (MSC) on the progression of IBD. Methods: In this study, human bone marrow-derived MSCs were injected into IL-10 KO mice (MSC). Oxidative stress and inflammation levels were evaluated in the large intestine and compared with those in control IL-10 KO mice (CON) and normal wild-type control mice (Wild). Results: The levels of ROS (superoxide and hydrogen peroxidase) and a secondary end-product of lipid peroxidation (malondialdehyde) were considerably higher in the CON, while superoxide dismutase and catalase levels were lower in the MSC. Inflammation-related marker (interferon- $\gamma$, tumor necrosis factor- $\alpha$, IL-4, and CD8) expression and inflammatory histological changes were much less pronounced in MSC than in CON. Conclusions: MSCs affect the redox balance, leading to the suppression of IBD. (Gut Liver 2020;14:100-107)

Key Words: Inflammatory bowel diseases; Mesenchymal stem cells; Oxidative stress; Interleukin-10 knockout KO mouse

\section{INTRODUCTION}

Inflammatory bowel disease (IBD) is a gastrointestinal tract disease that manifests as Crohn's disease or ulcerative colitis and is characterized by chronic inflammation in the gut. The ethology of IBD still remains unknown but studies of its pathogenesis suggest that malfunction of the intestinal immune system and environmental risk factors, such as the gut microbiota, are important in this disease. ${ }^{1,2}$ The intestinal mucosa provides the primary defense barrier against gut microbiota, with high levels of inflammation triggering reactions in the body. Bacterial attack induces activation of the immune system, resulting in cross-talk between lymphocytes and antigen-presenting cells. Simultaneously, gut microbiota stimulate the epithelium and increase the secretion of chemokines and cytokines, which activate mucosal immune cells. ${ }^{3}$ However, it is unclear whether an abnormal immune response to a normal microbiota occurs or a normal immune response to abnormal microbiota occurs. ${ }^{4}$

In the past 20 years, numerous studies have identified specific factors contributing to IBD pathogenesis such as autophagy, barrier defence, ${ }^{7,8}$ and T-cell differentiation signalling, ${ }^{9,10}$ but a cure for IBD is lacking. Treatments for IBD involve symptom alleviation using anti-inflammatory agents such as steroids or removing a segment of the colon in the uncontrolled state. Although biological agents such as anti-tumor necrosis factor (anti-TNF) can be administered, $40 \%$ of patients show no response to this treatment. ${ }^{6}$

Reactive oxygen species (ROS) are released during normal metabolism and play an important role in biological regulation. Cells have developed anti-oxidizing agents such as catalase, superoxide dismutase (SOD), and glutathione to protect against ROS and their adverse effects. However, homeostasis of redox

\footnotetext{
Correspondence to: In Hwan Song

Department of Anatomy, Yeungnam University College of Medicine, 170 Hyeonchung-ro, Nam-gu, Daegu 42415, Korea

Tel: +82-53-640-6912, Fax: +82-53-621-5083, E-mail: ihsong@med.yu.ac.kr

Received on September 27, 2018. Revised on March 20, 2019. Accepted on March 29, 2019. Published online September $19,2019$. pISSN 1976-2283 eISSN 2005-1212 https://doi.org/10.5009/gnl18438

(a) This is an Open Access article distributed under the terms of the Creative Commons Attribution Non-Commercial License (http://creativecommons.org/licenses/by-nc/4.0) which permits unrestricted non-commercial use, distribution, and reproduction in any medium, provided the original work is properly cited.
} 
signaling is disrupted by excessive ROS production to result in cell injury. Increasing evidence from recent studies indicated that oxidative stress is associated with the pathophysiology of inflammation, cancer, diabetes mellitus, hypertension, ageing, and neurodegenerative disease. , $^{411}$

Mesenchymal stem cells (MSCs) have been identified in numerous organs including the bone marrow, adipose tissue, dental pulp, and skeletal muscle; moreover, MSC niches are associated with the micro-vasculature throughout the body in the form of pericytes. These cells can differentiate into diverse mesenchymal cell lineages and exhibit the potential to increase the regeneration of damaged tissue or organs. ${ }^{12}$ MSCs secrete cytokines and ameliorate the microenvironment for tissue regeneration. Additionally, MSCs have potent immunomodulatory effects by decreasing inflammatory cell proliferation and cytokine secretion from cells. ${ }^{13}$

Interleukin-10 (IL-10) is an anti-inflammatory cytokine mainly secreted by monocytes, lymphocytes, and mast cells. IL-10 has multiple effects on inflammation and immunomodulation by downregulating inflammatory cytokines. ${ }^{14}$ IL-10 knockout (KO) mice are a well-known animal model of IBD that develops spontaneous gut inflammation similar Crohn's disease. In this study, we administered MSCs to IL-10 KO mice and evaluated production of ROS and its scavengers, inflammatory cytokine expression, and histological findings. The results were compared to those from parallel control IL-10 KO (CON) and normal wildtype mice to evaluate the effects of MSCs on progress of IBD.

\section{MATERIALS AND METHODS}

\section{MSCs culture and characterization}

Human bone marrow samples were harvested from the iliac crest of the donors using a protocol approved by the Internal Review Board of Yeungnam University Hospital. MSCs were isolated using modified versions of previously described methods. ${ }^{15}$ Briefly, mononuclear cells were isolated using a density gradient centrifuge with density 1.08 Percoll (Sigma, St. Louis, MO, USA) solution at $480 \times g$ for 15 minutes. and were cultured in DMEM (Dulbecco Modified Eagle Medium; Gibco-BRL, Grand Island, NY, USA) with 10\% fetal bovine serum (FBS; GibcoBRL), penicillin, and streptomycin (Gibco-BRL). The cells from passages 2-3 were used in this study.

For the following cytometric analysis, the cells were trypsinized and incubated with primary antibodies for mouse antihuman CD105 (Abcam, Cambridge, UK), mouse anti-human CD90 (Abcam), mouse anti-human CD73 (Abcam), and mouse anti-human CD45 (Abcam) for 30 minutes at $25^{\circ} \mathrm{C}$, while no antibodies were included in the negative control. After incubation with secondary fluorescein isothiocyanate-labelled antimouse IgG (Sigma) in the dark for 30 minutes, the cells were analyzed with a FACScan fluorescence-activated cell sorter (BD Biosciences, Franklin Lakes, NJ, USA) equipped with CellQuest software (BD Biosciences).

To evaluate the differentiation potential, the cells were incubated in osteogenic and adipogenic media for 1 week, and Oil red 0 (Sigma) and ELF97 ${ }^{\circledR}$ Endogenous Phosphatase Detection Kit (Molecular Probes, Eugene, OR, USA) were used to stain lipid droplets in adipocytes and alkaline phosphatase in osteoblasts, respectively. ${ }^{15}$

\section{Animals and procedures}

Eleven-week-old wild C57BL/6 and IL-10 ${ }^{-1-}$ mice were obtained from Koatech (Pyeongtaek, Korea) and Jackson Laboratories (Bar Harbor, ME, USA), respectively. The mice were maintained in specific pathogen free condition and all experimental protocols were approved by the Institutional Animal Care and Use Committee of College of Medicine, Yeungnam University. $\mathrm{IL}-10^{-/-}$mice were used for MSC and CON groups ( $\mathrm{n}=6$ each) whereas normal wild type C57BL/6 mice were used for Wild group $(n=6)$ and adapted while feeding of a normal rodent chew diet. After 1 week, animals were divided into Wild, MSC, and CON groups and adapted while feeding of a normal rodent chew diet. After 1 week, $200 \mu \mathrm{L}$ PBS containing $5 \times 10^{5}$ MSCs were injected through the tail vein for the MSC group and the same volume of PBS was used for the CON and Wild groups. One week after cell injection, another booster injection of MSCs or PBS was administered. One week after booster injection, the animals were sacrificed, and the large intestines were harvested. Tissue samples were divided into the cecum, ascending colon, and descending colon. Part of the sample was fixed in 3.7\% paraformaldehyde overnight at $4^{\circ} \mathrm{C}$, dehydrated in ethanol, and embedded in paraffin. The sections were stained with hematoxylin and eosin for observation of general histological changes or Manson's trichrome, collagen fiber specific stain, for evaluation of collagen deposition. The remaining tissues were frozen in liquid nitrogen for molecular biological analysis.

\section{Oxidative stress analysis}

To measure superoxide $\left(\mathrm{O}_{2}^{-}\right)$levels, $20 \mu \mathrm{L}$ of tissue lysates and $200 \mu \mathrm{L}$ of dihydroethidium (10 $\mu \mathrm{M}$; Sigma) were incubated in 96 -well plates for 10 minutes at $25^{\circ} \mathrm{C}$. Fluorescence was measured with a Spectra Max Gemini EM fluorescence microplate reader (Molecular Devices, Sunnyvale, CA, USA) at excitation/ emission wavelengths of $544 / 612 \mathrm{~nm}$ at $37^{\circ} \mathrm{C}$. The relative fluorescence intensity versus the Wild group were reported as data. Xylenol orange (Sigma) was used for measuring hydrogen peroxide $\left(\mathrm{H}_{2} \mathrm{O}_{2}\right)$ levels. One hundred microliters of tissue lysate (100 $\mu \mathrm{g}$ protein) were incubated for 30 minutes at $25^{\circ} \mathrm{C}$ with $900 \mu \mathrm{L}$ of Fox reagent ( $25 \mathrm{mM} \mathrm{H}_{2} \mathrm{SO}_{4}, 0.1 \mathrm{M}$ sorbitol, $2.5 \mathrm{mM}$ ferrous ammonium sulphate, and $0.1 \mathrm{mM}$ xylenol orange). Supernatants was collected and measured absorbance using an E max Precision Microplate Reader (Molecular Devices) ${ }^{16}$ at $570 \mathrm{~nm}$. Lipid peroxidation levels were evaluated by measuring the formation of malondialdehyde (MDA). One hundred microliters of tissue 
lysate (100 $\mu$ g protein) were mixed with $1.4 \mathrm{~mL}$ of TBARS solution (0.375\% thiobarbituric acid, 15\% trichloroacetic acid, and $0.25 \mathrm{~N} \mathrm{HCl})$ and then heated in boiling water $\left(95^{\circ} \mathrm{C}\right.$ to $\left.100^{\circ} \mathrm{C}\right)$ for 15 minutes to develop the pink color. After centrifugation at $13,000 \times \mathrm{g}$ for 10 minutes, supernatants were collected and absorption was measured using an E max Precision Microplate Reader (Molecular Devices) ${ }^{17}$ at $540 \mathrm{~nm}$.

\section{Western blot analysis}

For Western blotting experiments, protein electrophoresis was performed as described previously. ${ }^{17}$ Proteins were transferred onto Immobilon-P membranes (Millipore, Billerica, MA, USA), blocked with 5\% skim milk in Tris buffered saline with Tween 20 (TBST), and incubated overnight in $25^{\circ} \mathrm{C}$ with the following antibodies against various proteins: CD4 (1:1,000; Novus Biologicals, Littleton, CO, USA), CD8 (1:1,000; Novus Biologicals), TNF- $\alpha$ (1:1,000; Abcam), interferon (INF)- $\gamma$ (1:1,000; Abcam), IL-4 (1:1,000; Abcam), phospho (p)-NF-кB/p65 (1:1,000; Cell Signaling, Danvers, MA, USA), SOD 1 (1:1,000; Enzo Life Sciences, Farmingdale, NY, USA), SOD2 (1:1,000; Bioworld Technology, Inc., St. Louis Park, MN, USA), catalase (1:1,000; Bioworld Technology, Inc), fibronectin (1:1,000; BD Biosciences), type I collagen (1:1,000; Abcam), and $\beta$-actin (1:5,000; Santa Cruz Biotechnology, Dallas, TX, USA). After washing, the mem- branes were incubated for 1 hour at $25^{\circ} \mathrm{C}$ with a $1: 700$ dilution of horseradish peroxidase-conjugated horse anti-mouse (Santa Cruz Biotechnology), anti-goat (Santa Cruz Biotechnology), or goat anti-rabbit (Santa Cruz Biotechnology) antibody. The specific proteins were visualized by enhanced chemiluminescence (Millipore). $\beta$-Actin was used as a loading control for normalization of the respective proteins. Band density was quantified using ImageJ software (National Institutes of Health, Bethesda, MD, USA).

\section{Statistical analysis}

All graphs are figured as the mean \pm standard error. One-way analysis of variance was performed to assess statistical differences between groups. Differences between groups were considered statistically significant at $\mathrm{p}<0.05$.

\section{RESULTS}

\section{MSC characteristics}

Culture dish-attached MSCs grew rapidly and showed the typical shape of MSCs as polygonal with multiple cytoplasmic processes. Flow cytometric analysis revealed no expression of the leukocyte marker CD45 or hematopoietic cell marker CD34, as in the negative control. The MSC markers CD73, CD90, and
A
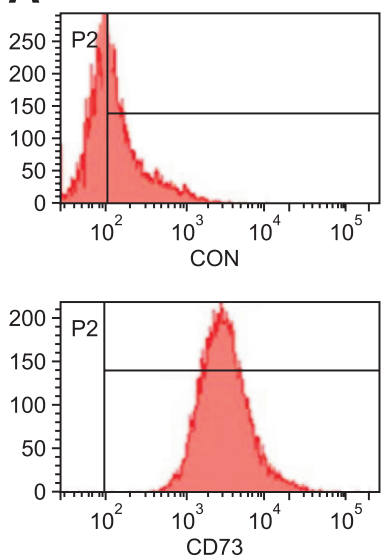
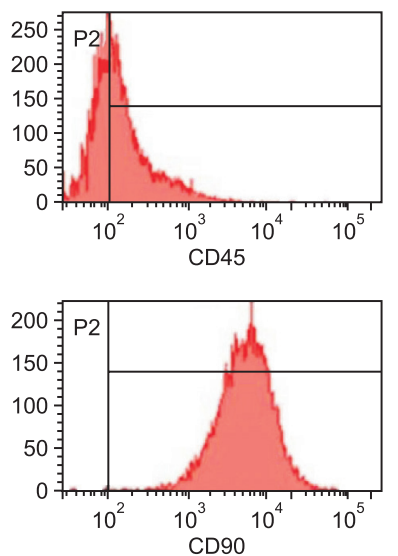
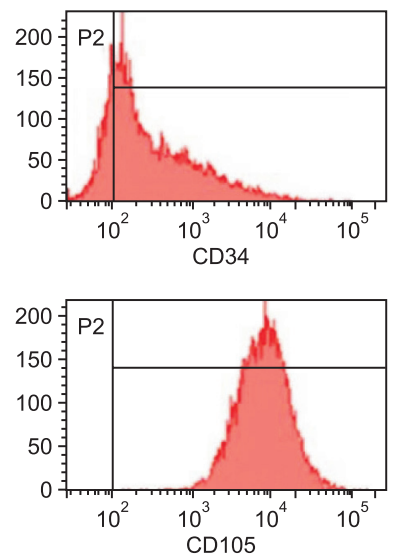
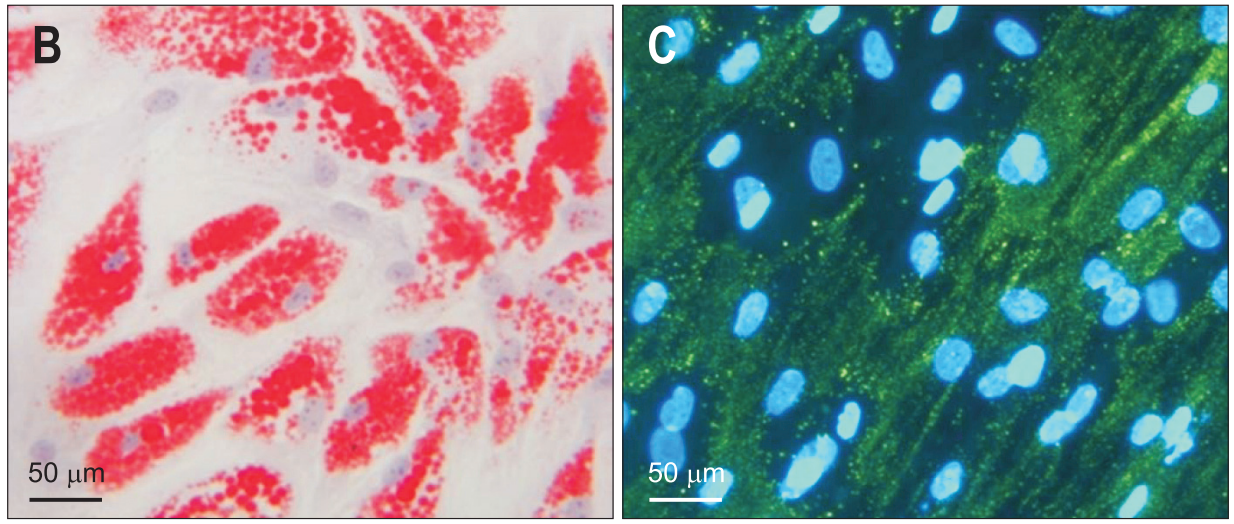

Fig. 1. Stem cell characterization. Flow cytometric analysis of surface $\mathrm{CD}$ marker expression in cultured mesenchymal stem cell (MSC). MSC markers (CD73, CD90, and CD105) were highly expressed, while a hematopoietic marker (CD34) and a leukocyte marker (CD45) were not expressed (A). MSCs cultured in adipogenic media contained Oil Red 0-stained lipid droplets in the cytoplasm (B), while MSCs cultured in osteogenic media possessed ELFstained alkaline phosphatase granules in the cytoplasm (C). CON, control. 
CD105 were highly expressed (Fig. 1A). The plasticity of MSCs was detected as their differentiation into adipocytes and osteoblasts. Abundant round red stained lipid droplets were observed in the cells cultured in adipogenic media (Fig. 1B) and intensive fluorescent dots, stained by ELF for alkaline phosphatase, were observed in cells cultured in osteogenic media (Fig. 1C).

\section{Gross and microscopic findings}

Compared to the transparent pinkish wild-type intestine, the wall of IL-10 K0 mouse intestine was thick and whitish opaque. This pattern was milder in the MSC group than in the CON group. The solidity of the fecal material caused the intestine to form a regular bolus shape in the Wild group, but this effect disappeared in the CON group because of diarrhea in IL-10 KO mice. Lesser bolus formation was observed in the CON group compared to in the MSC group (Fig. 2).

Mucosal and submucosal thickening and inflammatory cell infiltration were clearly observed in IL-10 KO mice. The infil- tration of the cell was prominent in the lamina propria. Such inflammatory changes were severe in the CON group compared to in the MSC group (Fig. 3).

\section{Inflammation and fibrosis}

The number of the cells expressing helper-T cell marker (CD4) was increased in MSC and CON compared to Wild but no difference was observed between MSC and CON groups. Killer-T cell marker (CD8) expression was lower in the MSC group than in the CON group. Inflammation-related gene (TNF- $\alpha$, INF- $\gamma$, IL-4, and $\mathrm{p}-\mathrm{NF}-\mathrm{kB}$ ) expression was also significantly elevated in the CON group compared to in the MSC group (Fig. 4).

Fibrosis-related protein (fibronectin and type I collagen) expression was increased in IL-10 K0 mice, and type I collagen level was significantly higher than in the MSC group (Fig. 5). Manson's trichrome stained blue-colored collagen fibers were observed predominantly in the submucosal layer and the staining intensity was correlated with the results of Western blotting,
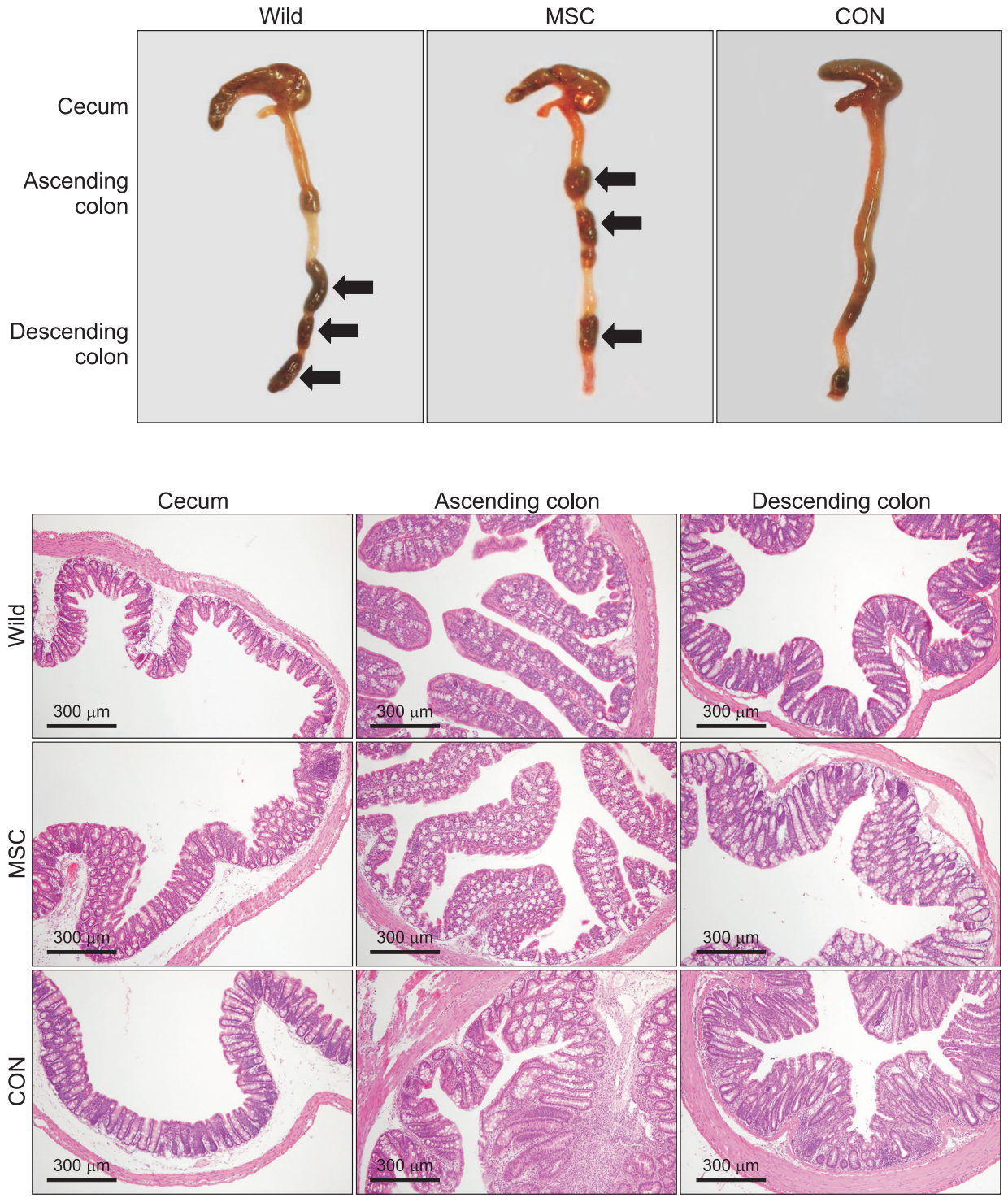

Fig. 2. Gross observation of cecum, ascending colon and descending colon of the normal wild-type control mice (Wild) and saline-treated (CON) or MSCs-treated (MSC) IL-10 KO mice. Intestinal wall thickening was obvious in IL-10 K0 mice, while bolus formation owing to the solidity of fecal material (arrows) was more prominent in the MSC group than in the CON group.

MSCs, mesenchymal stem cells; IL$10 \mathrm{KO}$, interleukin-10 knockout.
Fig. 3. Photomicrographs of the cecum, ascending colon and descending colon of the normal wildtype control mice (Wild) and MSCstreated (MSC) or saline-treated (CON) IL-10 KO mice. Inflammatory cell infiltration and lamina propria thickening were prominent in the CON group (H\&E).

MSCs, mesenchymal stem cells; IL$10 \mathrm{KO}$, interleukin-10 knockout. 

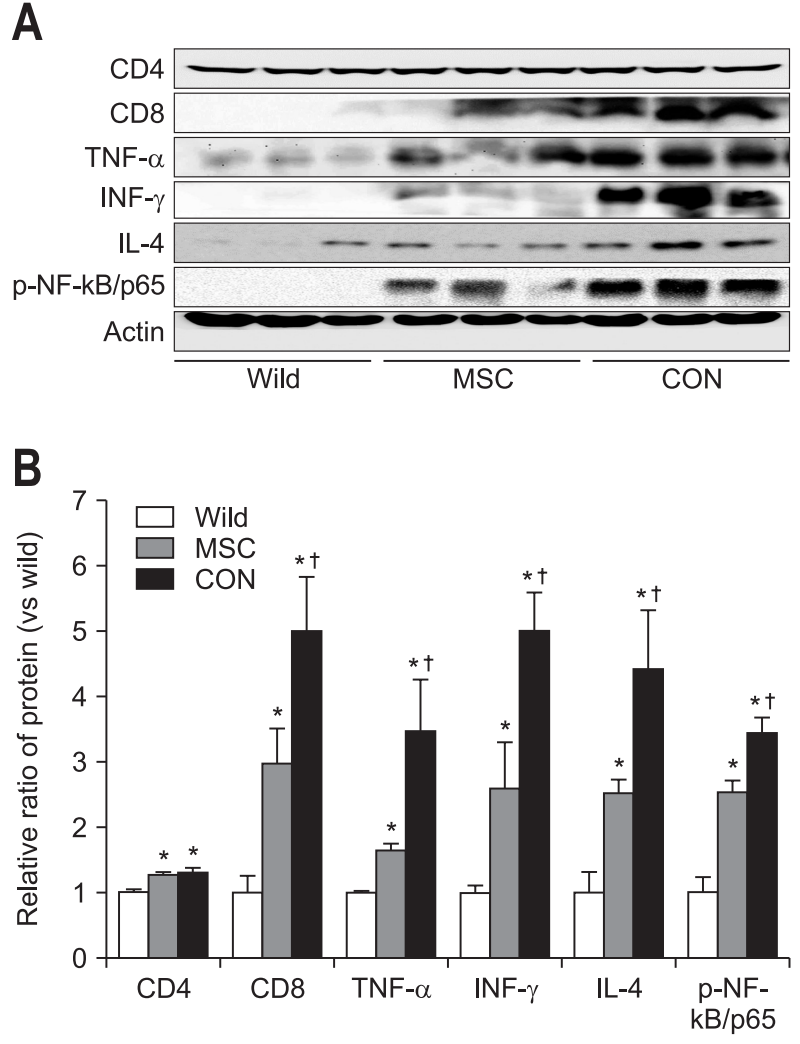

Fig. 4. (A) Expression levels of inflammation-related genes in the normal wild-type control mice (Wild) and MSCs-treated (MSC) or saline-treated (CON) IL-10 KO mice. (B) Bands were standardized and are shown in the graph. CD4, CD8, TNF- $\alpha$, INF- $\gamma$, IL-4, and p-NF- $\kappa \mathrm{B} /$ p65 expression levels were significantly lower in the MSC group than in the CON group. Bars show mean \pm SE.

MSC, mesenchymal stem cell; TNF- $\alpha$, tumor necrosis factor $\alpha$; INF- $\gamma$,

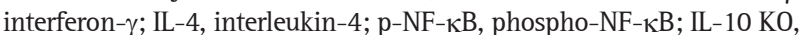
interleukin-10 knockout; SE, standard error. ${ }^{*} \mathrm{p}<0.05$ vs Wild; ${ }^{\dagger} \mathrm{p}<0.05$ vs in MSC $(n=6)$.

particularly in the cecum and ascending colon (Fig. 6).

\section{Oxidative stress}

ROS (superoxide and hydrogen peroxide) and lipid MDA formation was significantly higher in the CON group than in the MSC and Wild groups (Fig. 7). Expression of antioxidant enzymes showed the reverse pattern as oxidative stress. Catalase and SOD1 formation was decreased in IL-10 KO mice but was significantly higher in the MSC group than in the CON group, whereas SDO2 showed similar levels (Fig. 8).

\section{DISCUSSION}

Despite intensive research in the last two decades, the exact etiology of IBD remains unclear. A combination of a patient's immune response, microbiota, environment, and genetics play an important role in the induction and aggravation of the inflammatory cascade in patients with Crohn's disease and those with ulcerative colitis. ${ }^{18}$ Because IBD is an interactive disease
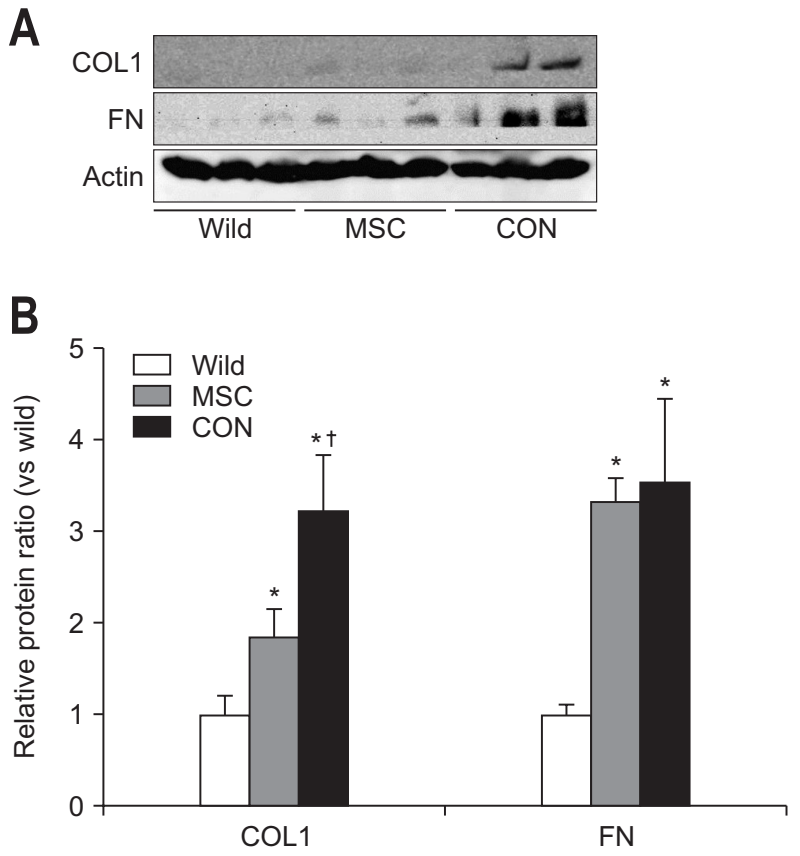

Fig. 5. (A) Fibrosis-related protein expression level in the normal wild-type control mice (Wild) and MSCs-treated (MSC) or salinetreated (CON) IL-10 KO mice. (B) The level of type I collagen was elevated in IL-10 KO mice, and the CON group showed significantly higher levels than the MSC group. Bars show the mean \pm SE. MSC, mesenchymal stem cell; IL-10 KO, interleukin-10 knockout; $\mathrm{SE}$, standard error, FN, fibronectin; COL1, type I collagen. * $\mathrm{p}<0.05$ vs Wild; $\uparrow \mathrm{p}<0.05$ vs in MSC $(n=6)$.

with predisposing inherent factors, unidirectional chemical or biological treatment focusing on wound healing or immunological abnormalities may not produce satisfactory results. MSCs introduced as multipotent mesodermal stem cells can be used as a cell source for tissue healing in regenerative medicine. ${ }^{19}$ Numerous studies altered the concept of MSCs to "drug stores" or "medicinal signaling cells" because bioactive factors from exogenously supplied MSCs suppress over-activation of the immune system and stimulate the patient's site-specific and tissue-specific resident stem cells to construct new tissue. ${ }^{20,21}$ The multiple biological functions of stem cells may be applicable in cell-based therapy. However, optimization of MSC therapy appears to be the most important factor and can be resolved only by determining the mechanisms of therapeutic functions. However, the translational arm of stem cell biology requires further development. ${ }^{21}$ We previously reported that MSCs attenuate IBD by improving histological disturbances such as inflammation and decrease inflammatory cytokine expression. ${ }^{22}$ This result provided the basis for our further studies of the mechanism of MSCs in IBD.

Included in our previous study, piroxicam is frequently used to induce reliable IBD induction in IL-10 KO mouse models. In this study, we used 12-week-old mice after a preliminary study of reliable IBD development without piroxicam because piroxicam influences oxidative stress. ${ }^{23}$ Gross and histological changes 


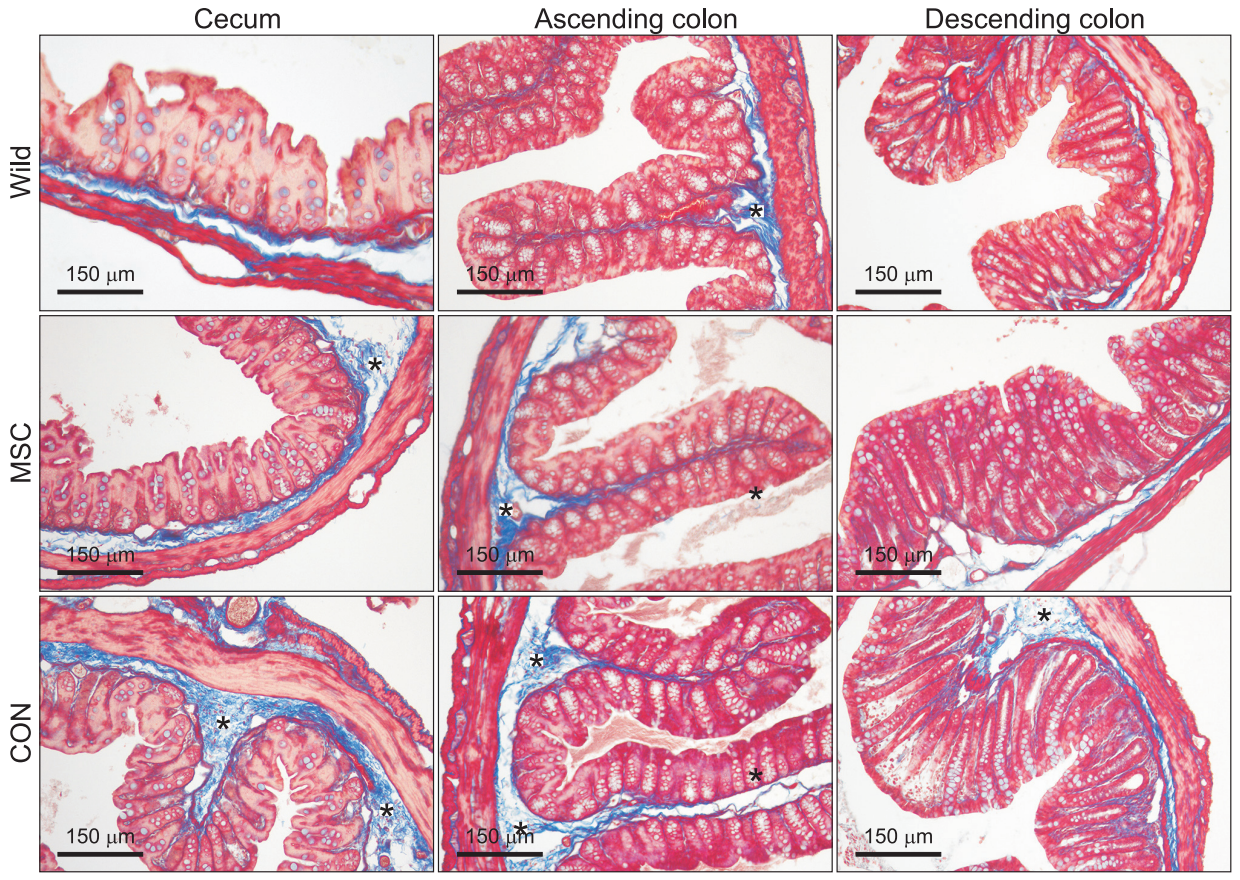

Fig. 6. Collagen deposition in the deposition in the normal wild-type control (Wild) and MSCs-treated (MSC) or saline-treated (CON) IL10 KO mice. Manson's trichromestained blue-colored collagen fibers (*) were observed predominantly in the submucosal layer, and the intensity of staining was correlated with the Western blot results, particularly in the cecum and ascending colon. MSC, mesenchymal stem cell; IL-10 KO, interleukin-10 knockout.
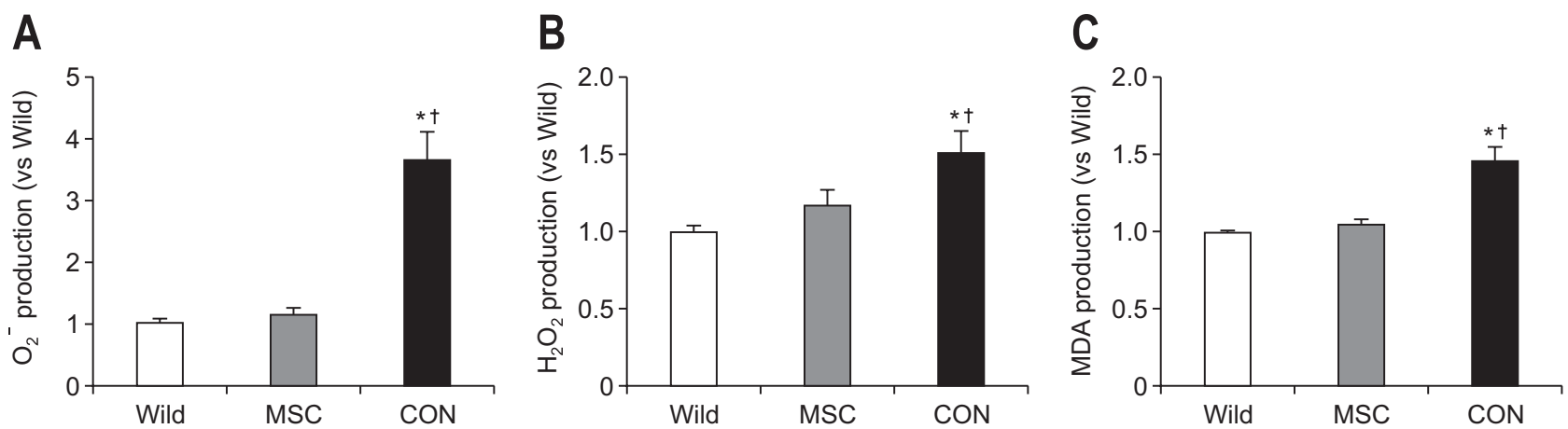

Fig. 7. Production levels of superoxide (A), hydrogen peroxide (B), and MDA (C) in the large intestine, which were significantly higher in the saline-treated (CON) group than in the normal wild-type control (Wild) and MSCs-treated (MSC) groups. Bars show the mean \pm SE. MDA, malondialdehyde; MSC, mesenchymal stem cell; SE, standard error. ${ }^{*} \mathrm{p}<0.05$ vs Wild; $\dagger \mathrm{p}<0.05$ vs in MSC ( $\mathrm{n}=6$ ).

as well as inflammatory cytokine expression were severe in the CON group compared to in the MSC group in this study model. Our data showed that ROS (superoxide and hydrogen peroxide) and MDA, the end-product of lipid oxidation, were significantly reduced in the MSC group compared to in the CON group. Decreased ROS scavenger (catalase and SOD1) levels in IL-10 KO mice (CON) were preserved in MSC-transferred mice (MSC). The slight change in mitochondrial SOD (SOD2) compared to cytoplasmic SOD (SOD1) and catalase suggests that MSCs influence cytoplasmic antioxidation.

Detailed studies in the past decade have shown that oxidative stress is one of major mechanism involved in the pathophysiology of IBD, ${ }^{24}$ particularly because an inflammatory process induces oxidative stress and reduces cellular antioxidant capacity and oxidative stress while aggravating inflammation. ${ }^{25,26}$ ROS play a dual role: they are essential for several processes in the cell, including intracellular signaling and regulatory mechanisms or host defense against microbiota at low levels, but damage organelles at high levels. Antioxidants play a predominant role in protecting against ROS. These molecules protect cells against pro-oxidant molecules by preventing or slowing the oxidation of other molecules. Various endogenous antioxidants in the cell are enzymes such as SOD, catalase, and glutathione peroxidase. A state in which increased formation of ROS overwhelms the body's antioxidant protection can be dangerous, as it may alter inflammatory responses and lead to lipid and protein modifications, DNA damage, apoptosis, or cancerogenic cell transformation. Thus, oxidative stress has been implicated in numerous human diseases, including IBD and colorectal cancer. ${ }^{27}$

Our data showed that MSCs decreased oxidative stress by reducing ROS production and by increasing antioxidant formation. Moreover, fibrosis in the intestine was mitigated by MSCs. 


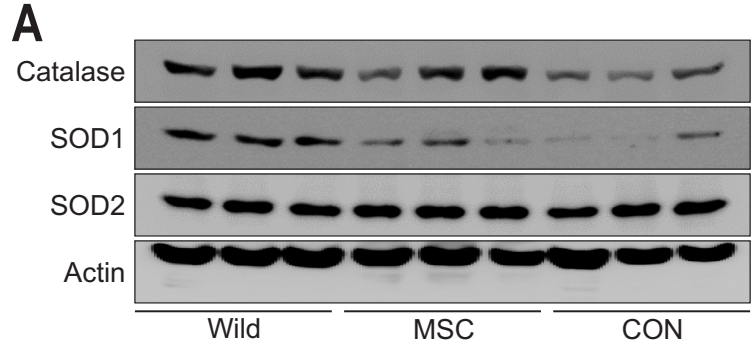

B

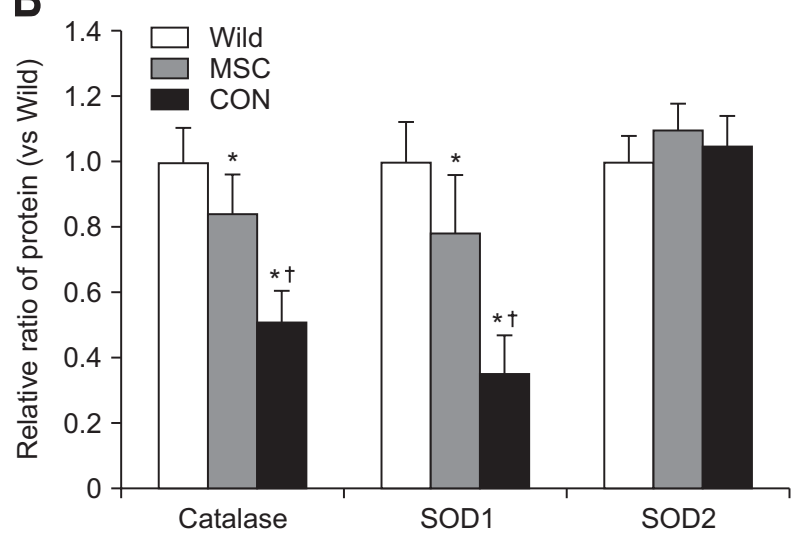

Fig. 8. Expression levels of superoxide dismutase (SOD) 1, 2 and catalase in the normal wild-type control (Wild) and MSCs-treated (MSC) or saline-treated (CON) groups (A). Bands were standardized and are shown in the graph (B). Catalase and SOD1 expression levels were lower in IL-10 KO mice than in Wild mice, but MSC treatment reduced the difference. Bars show the mean $\pm \mathrm{SE}$.

MSC, mesenchymal stem cell; IL-10 KO, interleukin-10 knockout; SE, standard error. ${ }^{*} \mathrm{p}<0.05$ vs Wild; ${ }^{\dagger} \mathrm{p}<0.05$ vs in MSC $(\mathrm{n}=6)$.

Inflammation and ROS are correlated and show synergic effects on tissue damage. Both drive apoptosis and fibrosis. Uncontrolled conditions can lead to irreversible tissue damage and result in organ failure. Thus, MSCs are promising candidates for the treatment of IBD because of their ability to reduce oxidative stress.

\section{CONFLICTS OF INTEREST}

No potential conflict of interest relevant to this article was reported.

\section{ACKNOWLEDGEMENTS}

This work was supported by the National Research Foundation of Korea Grant funded by the Korean Government (Ministry of Education; NRF-217C000377). This study was supported by 2016 Yeungnam University Grant.

\section{AUTHOR CONTRIBUTIONS}

Animal surgery, molecular work, data analysis: K.J.J. Molecu- lar work: T.J.L., S.Y.K. Bone marrow preparation: G.W.L., C.H.P. Histological analysis: J.Y.K., E.G.S. Study concept: B.I.J. Study design and interpretation: I.H.S.

\section{ORCID}

Kyong Jin Jung Gun Woo Lee Chul Hyun Park Tae Jin Lee Joo Young Kim Eon Gi Sung Seong Yong Kim Byung Ik Jang In Hwan Song https://orcid.org/0000-0002-5767-8002 https://orcid.org/0000-0002-8441-0802 https://orcid.org/0000-0002-3101-8655 https://orcid.org/0000-0003-3934-2035 https://orcid.org/0000-0002-0968-4504 https://orcid.org/0000-0003-1319-3852 https://orcid.org/0000-0003-3419-2699 https://orcid.org/0000-0002-3037-9272 https://orcid.org/0000-0002-0573-1836

\section{REFERENCES}

1. Sheehan D, Shanahan F. The gut microbiota in inflammatory bowel disease. Gastroenterol Clin North Am 2017;46:143-154.

2. de Lange KM, Barrett JC. Understanding inflammatory bowel disease via immunogenetics. J Autoimmun 2015;64:91-100.

3. Singh UP, Singh S, Singh R, Cong Y, Taub DD, Lillard JW Jr. CXCL10-producing mucosal CD4+ T cells, NK cells, and NKT cells are associated with chronic colitis in IL-10(-/-) mice, which can be abrogated by anti-CXCL10 antibody inhibition. J Interferon Cytokine Res 2008;28:31-43.

4. Sheehan D, Moran C, Shanahan F. The microbiota in inflammatory bowel disease. J Gastroenterol 2015;50:495-507.

5. Randall-Demllo S, Chieppa M, Eri R. Intestinal epithelium and autophagy: partners in gut homeostasis. Front Immunol 2013;4:301.

6. Pott J, Maloy KJ. Epithelial autophagy controls chronic colitis by reducing TNF-induced apoptosis. Autophagy 2018;14:1460-1461.

7. Sturm A, Dignass AU. Epithelial restitution and wound healing in inflammatory bowel disease. World J Gastroenterol 2008;14:348353.

8. van der Gracht E, Zahner S, Kronenberg M. When insult is added to injury: cross talk between ILCs and intestinal epithelium in IBD. Mediators Inflamm 2016;2016:9765238.

9. Zhernakova A, van Diemen CC, Wijmenga C. Detecting shared pathogenesis from the shared genetics of immune-related diseases. Nat Rev Genet 2009;10:43-55.

10. Smids C, Horjus Talabur Horje CS, Drylewicz J, et al. Intestinal $\mathrm{T}$ cell profiling in inflammatory bowel disease: linking $\mathrm{T}$ cell subsets to disease activity and disease course. J Crohns Colitis 2018;12:465-475.

11. Chakrabarti S, Jahandideh F, Wu J. Food-derived bioactive peptides on inflammation and oxidative stress. Biomed Res Int 2014;2014:608979.

12. Caplan AI. New MSC: MSCs as pericytes are Sentinels and gatekeepers. J Orthop Res 2017;35:1151-1159.

13. Zhao S, Wehner R, Bornhäuser M, Wassmuth R, Bachmann M, 
Schmitz M. Immunomodulatory properties of mesenchymal stromal cells and their therapeutic consequences for immune-mediated disorders. Stem Cells Dev 2010;19:607-614.

14. Nakagome K, Dohi M, Okunishi K, et al. In vivo IL-10 gene delivery suppresses airway eosinophilia and hyperreactivity by downregulating APC functions and migration without impairing the antigen-specific systemic immune response in a mouse model of allergic airway inflammation. J Immunol 2005;174:6955-6966.

15. Song IH, Dennis JE. Simple evaluation method for osteoinductive capacity of cells or scaffolds using ceramic cubes. Tissue Cell 2014;46:372-378.

16. Nourooz-Zadeh J. Ferrous ion oxidation in presence of xylenol orange for detection of lipid hydroperoxides in plasma. Methods Enzymol 1999;300:58-62.

17. Song IH, Jung KJ, Lee TJ, et al. Mesenchymal stem cells attenuate adriamycin-induced nephropathy by diminishing oxidative stress and inflammation via downregulation of the NF-kappaB. Nephrology (Carlton) 2018;23:483-492.

18. Baumgart DC, Carding SR. Inflammatory bowel disease: cause and immunobiology. Lancet 2007;369:1627-1640.

19. Song IH. Adult mesenchymal stem cells for cell therapy in clinical application. Yeungnam Univ J Med 2009;26:1-14.
20. Caplan AI. Mesenchymal stem cells: time to change the name! Stem Cells Transl Med 2017;6:1445-1451.

21. Nagaishi K, Arimura Y, Fujimiya M. Stem cell therapy for inflammatory bowel disease. J Gastroenterol 2015;50:280-286.

22. Jung WY, Kang JH, Kim KG, et al. Human adipose-derived stem cells attenuate inflammatory bowel disease in IL-10 knockout mice. Tissue Cell 2015;47:86-93.

23. Montalvo-Javé EE, Ortega-Salgado JA, Castell A, et al. Piroxicam and meloxicam ameliorate hepatic oxidative stress and protein carbonylation in Kupffer and sinusoidal endothelial cells promoted by ischemia-reperfusion injury. Transpl Int 2011;24:489-500.

24. Weber C. IBD: Lactococcus lactis alleviates oxidative stress and colitis in mice. Nat Rev Gastroenterol Hepatol 2015;12:429.

25. Halliwell B, Gutteridge JM. Role of free radicals and catalytic metal ions in human disease: an overview. Methods Enzymol 1990;186:1-85.

26. Jomova K, Valko M. Advances in metal-induced oxidative stress and human disease. Toxicology 2011;283:65-87.

27. Piechota-Polanczyk A, Fichna J. Review article: the role of oxidative stress in pathogenesis and treatment of inflammatory bowel diseases. Naunyn Schmiedebergs Arch Pharmacol 2014;387:605620. 